\title{
Experimental investigation on heat recovery from flue gas using falling film method
}

\author{
Chunhua Min ${ }^{\mathrm{a}, \mathrm{b}}$, Xuguang Yang ${ }^{\mathrm{a}, \mathrm{b}}$, Jing He $\mathrm{He}^{\mathrm{a}, \mathrm{b}}$, Kun Wang ${ }^{\mathrm{a}, \mathrm{b}}$, Liyao Xie ${ }^{\mathrm{a}, \mathrm{b}}$,
}

Daniel I. Onwude ${ }^{\mathrm{c}, \mathrm{d}}$, Wenbin Zhang ${ }^{\mathrm{e}}$, Hongwei $\mathrm{Wu}^{\mathrm{e} *}$

${ }^{a}$ School of Energy and Environmental Engineering, Hebei University of Technology, Tianjin 300401, P R China

${ }^{\mathrm{b}}$ Hebei Key Laboratory of Thermal Science and Energy Clean Utilization, Hebei University of Technology, Tianjin 300401, P R China

${ }^{\mathrm{c}}$ Empa, Swiss Federal Laboratories for Materials Science and Technology, Laboratory for Biomimetic Membranes and Textiles, Lerchenfeldstrasse 5, CH-9014 St. Gallen, Switzerland ${ }^{\mathrm{d}}$ Department of Agricultural and Food Engineering, Faculty of Engineering, University of Uyo, 52021, Uyo, Nigeria

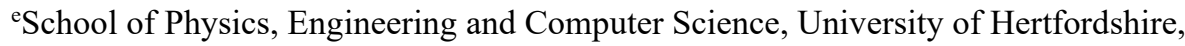
Hatfield, AL10 9AB, United Kingdom

*Corresponding Author (Dr. Hongwei Wu): Email: h.wu6@herts.ac.uk Tel: +44(0)1707284265

Abstract: The discharge of the flue gas is rich in water vapor and its latent heat, which leads to the main heat and moisture loss for the natural gas. In this article, based on the falling film spray technology, a new test rig was set up to test the heat and moisture recovery performance from flue gas. The effects of water-air ratio and inlet air temperature on the heat transfer effectiveness were examined in a systematic manner. Four case studies, i.e. empty tower, conical baffles staggered arranged in the tower, falling film plates and baffle plates fixed on the falling film plate were designed for the current system. Experimental results showed that: (i) increasing the water-air ratio or decreasing the inlet air temperature can improve the heat transfer effectiveness; (ii) the case with conical baffles shows the highest heat transfer effectiveness. In comparison with the falling film plates, the arranged baffle plates on the falling film plate can further improve the heat transfer effectiveness; (iii) for the cases with falling plates, the heat transfer effectiveness increases with the increase of the number of the falling film plates. The maximum heat transfer effectiveness is obtained at 5 falling film plates; (iv) for the 
case with conical baffles, increasing the floor of the conical baffles can improve the heat transfer effectiveness. In the range of the present work, the larger central angle of the spiracle is beneficial to increase the heat transfer effectiveness. For the optimal case in the range of the present work, the heat transfer effectiveness is $41.0 \%$ higher than that for the empty tower.

Keywords: falling film; spraying tower; heat recovery; flue gas; heat transfer

\section{Introduction}

As relatively clean energy with high quality and efficiency, natural gas has been widely used in power generation, petrochemical industry, mechanical manufacturing and other fields. It has been stated that China is expected to have a rapidly increasing natural gas consumer demand in the next few years $[1,2]$. As an important heating sector, natural gas-fired boiler has a thermal efficiency in the range of 70-80\% [3]. Generally, the boilers directly exhaust the flue gas at temperature of $150-250{ }^{\circ} \mathrm{C}$ to avoid dew-point corrosion. The discharge of the high-temperature flue gas is rich in water vapor and its latent heat accounts for more than $10 \%$ of the low calorific value of natural gas, which leads to the main heat loss of the boiler [4]. Therefore, waste heat recovery from the flue gas would be an effective way to improve the utilization efficiency of natural gas. On the other hand, recovering moisture from flue gas could promote clean production and saving water [5].

Typical methods for heat recovery from flue gas are normally to convert the waste heat into output work, heat pump and heat exchanger. Organic Rankine cycle [5-7] and 
Karina cycle $[8,9]$ are the advantageous power cycles for low-medium temperature heat recovery and have higher thermal efficiency in comparison with the steam power cycle. It has been reported that Karina cycle has the higher exergy efficiency in comparison with Organic Rankine cycle [10]. For the low temperature heat recovery, it is recognized that the heat pump or refrigeration system can use the waste heat for heating or cooling [11-13]. The most common technology for heat recovery is heat exchanger [14-16]. The enhancement technologies to improve the heat transfer of heat exchangers are often researched in the literatures [17]. Other technologies related to heat exchanger have been proposed and studied such as heat pipe $[18,19]$, regenerative heat exchanger [20], recuperative heat exchanger [21] and absorption heat exchange [22].

It is noted that when the working temperature is below dew point temperature, the above technologies can be used to moisture recovery from flue gas. However, the pipe with corrosion resistance should be used since the recovered water is acidic. The membrane method used for water and heat recovery can solve the above problem [23, 24].

Falling film evaporator has been widely applied in chemical industry and seawater desalination due to its high heat transfer performance and small heat transfer temperature difference [25-27]. In the present work, the falling film method is used for heat recovery from the flue gas by direct contacting between the water film and the flue gas. At the same time, the water vapor is absorbed by the water film with lower degree of acid. Hence, the acidic water can be relatively easily treated by alkalization. 
One can find that the heat pump or refrigeration system and heat exchanger are always used for low-temperature heat recovery. However, the heat pump or refrigeration system is complicated and expensive. The heat exchanger has the problem of low-temperature corrosion. Hence, a new method for heat recovery from lowtemperature flue gas is needed. According to the above discussions, the falling film method can fully or partly overcome the above problems and will be used for heat and moisture recovery in the present work. The effects of several control factors such as water-air ratio, inlet air temperature, number of falling plates, number of conical baffle floors and central angle spiracles punched in the water sprayer on the effect of heat recovery performance are experimentally studied in this paper.

\section{Experimental system and designs}

\subsection{Experimental system description}

The experimental system for heat recovery from flue gas using falling film spraying method is shown in Fig. 1. The wetted air is taken as the working medium to replace the flue gas in the present experimental study, which is a commonly used approach. For example, Zhu et al. [28] adopted a similar approach replacing the flue gas with the saturated wet air, and experimentally studied the flow and heat exchange process of saturated wet air-water in a simulated spray packed tower. In the present study, the air is wetted to saturated air in the bath heater prior to flowing into the spraying tower. The saturated air directly contacts with the liquid water in the tower. The latent heat and sensible heat of the saturated air are transferred to the spraying water. At the same time, the vapor in the air is condensed to the spraying water. The cooled saturated air enters 
the bath heater again from the tower. The heated spraying water and the condensed water flow back into the water tank.

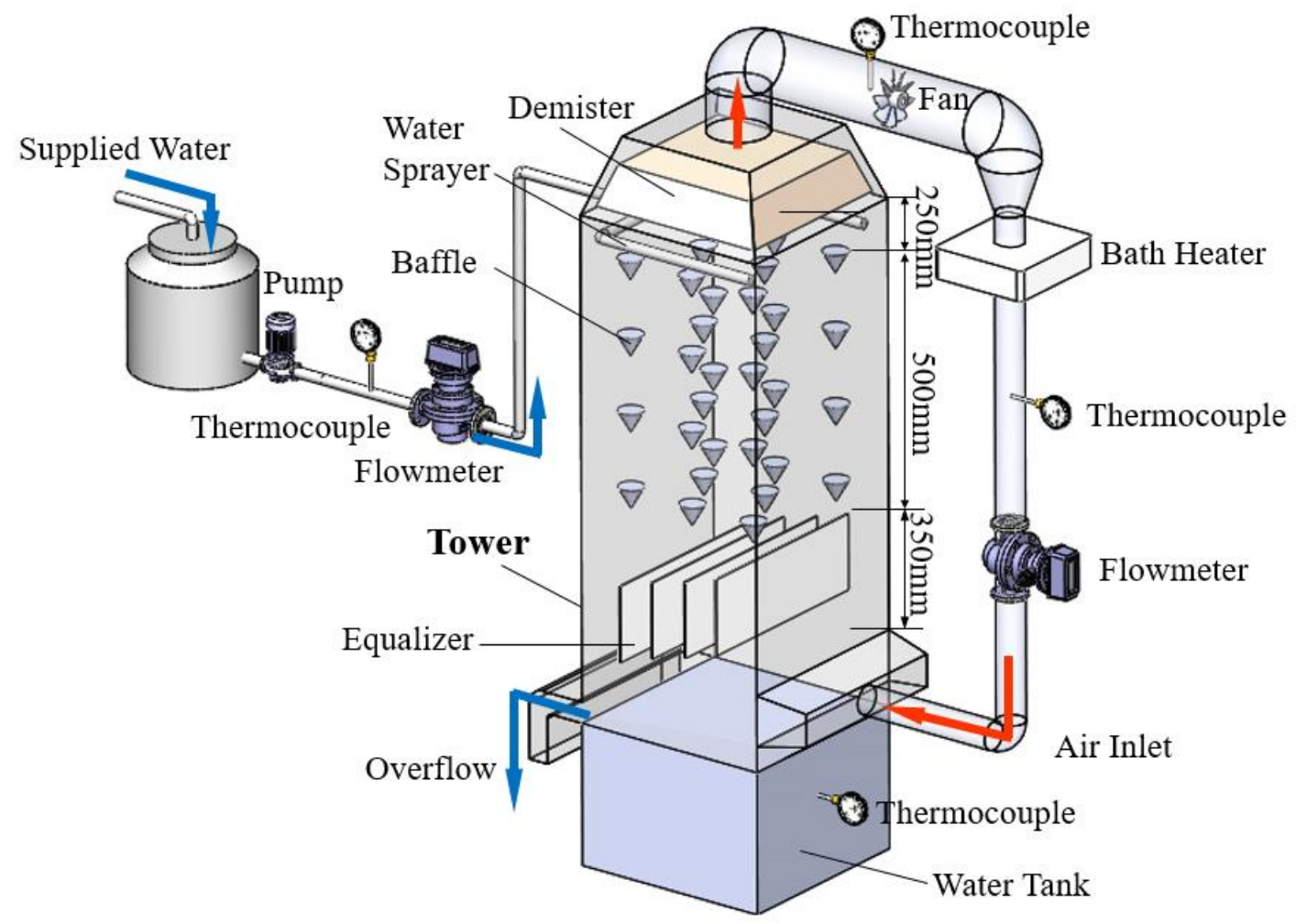

Fig. 1 Experimental system of falling film condensation heat transfer.

The tower has a rectangular section and the geometrical sizes are $200 \mathrm{~mm}$ in length $L, 200 \mathrm{~mm}$ in width $W$, and $1450 \mathrm{~mm}$ in height $H$, respectively. The outside surface of the tower is covered with rubber insulation material. The heat conduction is 0.034 $\mathrm{W} /(\mathrm{m} \cdot \mathrm{K})$ and the thickness is $20 \mathrm{~mm}$. The demisting domain, the spraying domain and the equalizing domain are $250 \mathrm{~mm}, 500 \mathrm{~mm}$ and $350 \mathrm{~mm}$ in height, respectively. Two water sprayers with $22 \mathrm{~mm}$ in diameter are fixed at the top of the spraying domain. Some staggered spiracles are punched in the water sprayer for spraying water downward, as shown in Fig. 2. The spiracle diameter $d$ and the pitch $p$ between two successive spiracles along the length direction are $1.5 \mathrm{~mm}$ and $10 \mathrm{~mm}$, respectively. There are three spiracles along the circumference and the central angle is marked as $\alpha$. 
As a reference design, $\alpha$ is selected as $30^{\circ}$. The flow rates of the saturated air and spraying water are controlled using converters. The temperature is measured using Ttype thermocouple and the uncertainty of the temperature is about $0.2 \mathrm{~K}$. The flow rate of the saturated air is measured using vortex flowmeter with accuracy of $\pm 4 \%$. The flow rate of the spraying water is measured using rotor flowmeter, its accuracy grade and measuring range are 1.5 and 40-400 L/h, respectively.

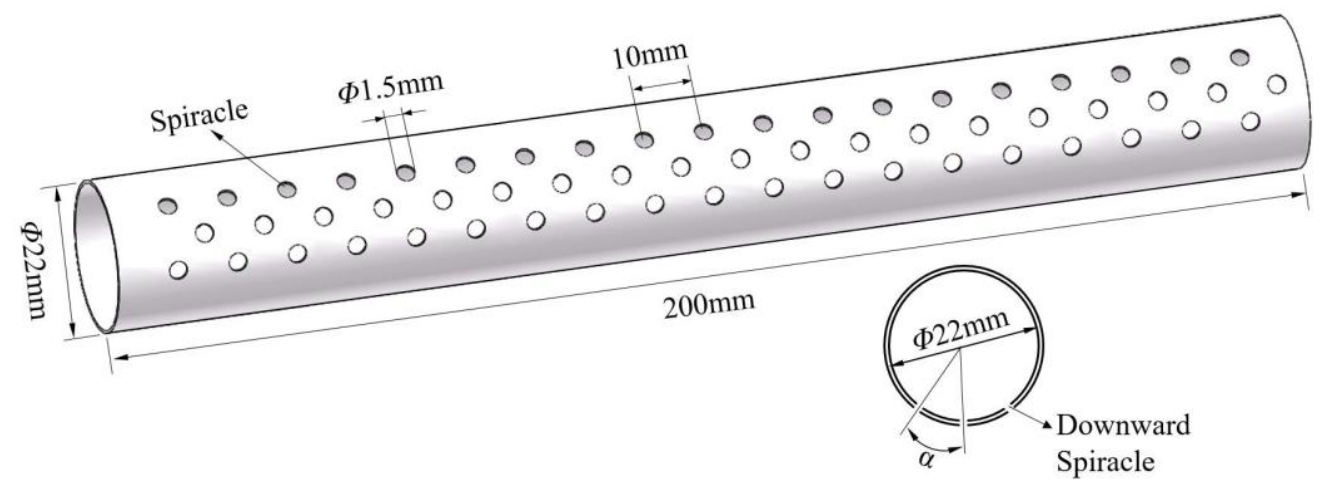

Fig. 2 Water sprayer with spiracles.

\subsection{Experimental designs}

Four different experimental cases will be investigated in the current work. The detailed designs are described as follows.

Case A: An empty tower for heat recovery is used. The spraying water drops downward and the wetted air flow upward in the tower. The wetted air directly contacts the spraying water and the heat and mass transfer occur in the tower.

Case B: Some conical baffles are staggered arranged in the tower. The arranged baffles can uniformly distribute the spraying water and the wetted air. At the same time, the contacting area and the contacting time between the spraying water and the wetted air increased due to the increased flow resistance. The bottom diameter and the height of the baffle are $50 \mathrm{~mm}$ and $43.3 \mathrm{~mm}$, respectively, as shown in Fig. 3. As a reference 
design for this case, 15 baffles are arranged in two floors in the tower. There are 8 baffles in the upper floor while 7 baffles in the lower floor. The distance between the two floors is $12 \mathrm{~mm}$.

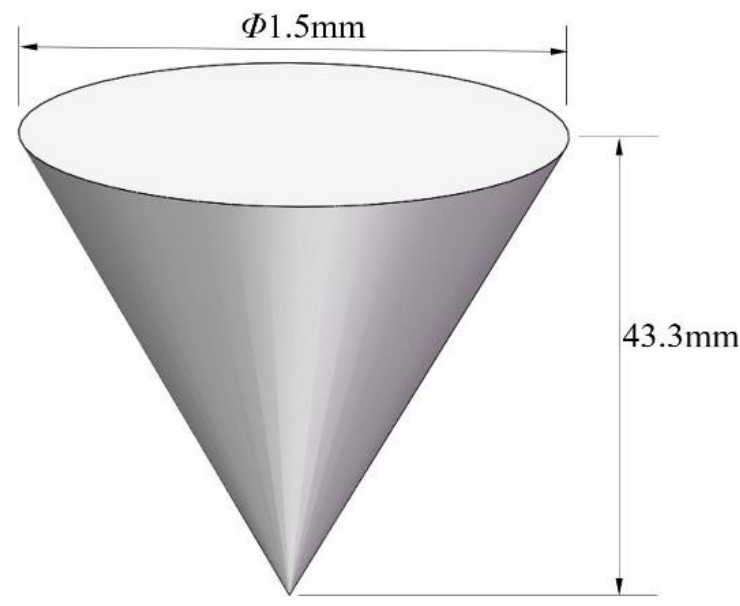

Fig. 3 A conical baffle.

Case C: Several falling film plates are arranged in the tower. In order to arrange the water film on the plate uniformly, several triangular water distributors are fixed at the top of the plates, as shown in Fig. 4. There are a total of seven plates in the tower. The thickness of each plate is $1 \mathrm{~mm}$. The edge length of the distributor is $10 \mathrm{~mm}$. As a base design, the plates number is $n_{\mathrm{p}}=7$. 


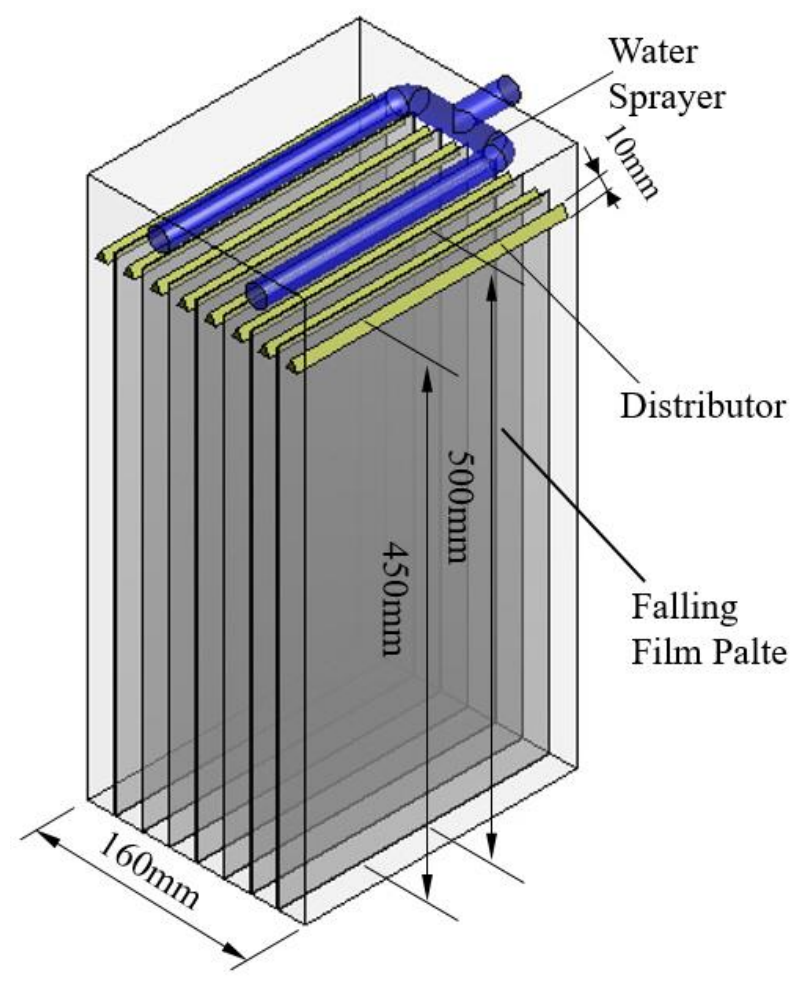

Fig. 4 Falling film plates in the tower.

Case D: Some baffle plates are fixed on the falling film plate, as shown in Fig. 5.

The angle $\theta$ between the baffle plate and the falling film plate is $30^{\circ}$. The width of the baffle plate $24 \mathrm{~mm}$.

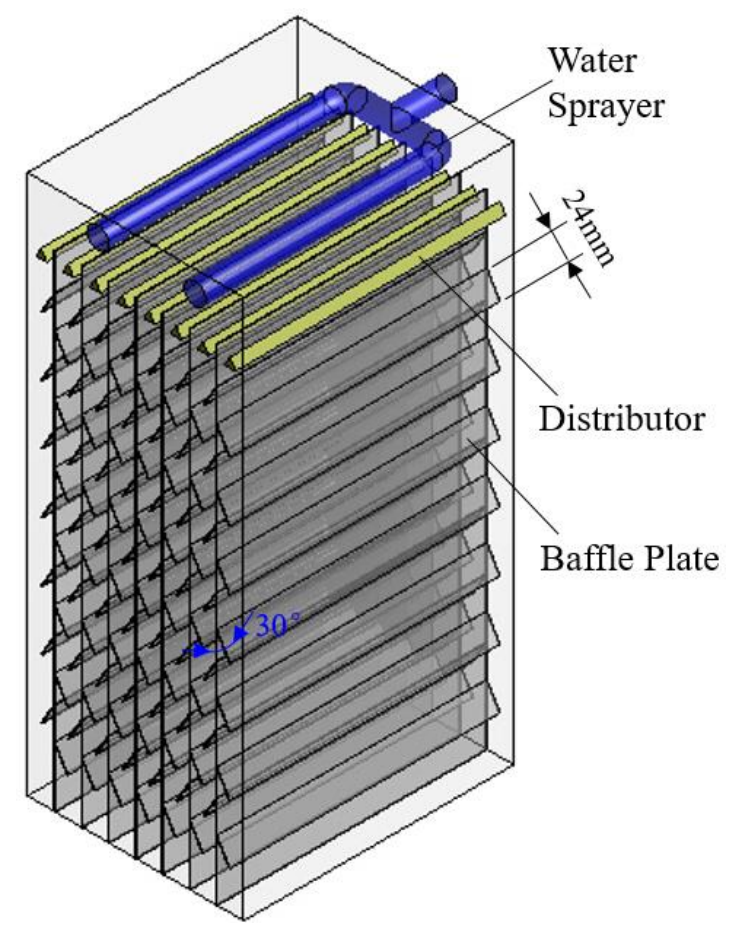

Fig. 5 Baffle plates on the falling film plate. 


\section{Data reduction}

To illustrate the heat transfer characteristics of the tower, the heat transfer effectiveness, $E$, is defined as:

$$
E=\frac{T_{\mathrm{a}, \text { in }}-T_{\mathrm{a}, \text { out }}}{T_{\mathrm{a}, \text { in }}-T_{\mathrm{w}, \text { in }}}
$$

where $T_{\mathrm{a} \text {,in }}, T_{\mathrm{a}, \text { out }}$ and $T_{\mathrm{w}, \text { in }}$ are the temperatures of the inlet air, outlet air and the inlet water, respectively.

The higher value of $E$ denotes the higher heat recovery performance and the higher heat transfer performance between the wetted air and the spraying water. It should be noted that both the inlet air and outlet air are saturated, thus, the heat transfer effectiveness can also reveal the moisture recovery from the wetted air. The higher value of $E$ also denotes the higher moisture recovery performance.

The water-air ratio, $r$, is defined as:

$$
r=\frac{\rho_{\mathrm{w}} Q_{\mathrm{v}, \mathrm{w}}}{\rho_{\mathrm{a}} Q_{\mathrm{v}, \mathrm{a}}}
$$

where $Q_{\mathrm{m}, \mathrm{w}}$ and $Q_{\mathrm{m}, \mathrm{w}}$ are the mass flow rates of the spraying water and wetted air, respectively.

The water and wetted air properties are assumed constant. The experimental uncertainty is estimated using the random uncertainty propagation technique [29]. If $F$ is a function of $n$ independent variables, each of which is marked as $X i$, the relative uncertainty of $F$ is given by:

$$
\frac{\delta F}{F}=\left[\sum_{i=1}^{n}\left(\frac{\delta X_{i}}{X_{i}}\right)^{2}\right]^{1 / 2}
$$

The value of the relative uncertainty for $E$ is computed by: 


$$
\frac{\delta E}{E}=\left[\frac{\delta T_{\mathrm{a}, \text { in }}^{2}+\delta T_{\mathrm{a}, \text { out }}^{2}}{\left(T_{\mathrm{a}, \text { in }}-T_{\mathrm{a}, \text { out }}\right)^{2}}+\frac{\delta T_{\mathrm{a}, \text { in }}^{2}+\delta T_{\mathrm{w}, \text { in }}^{2}}{\left(T_{\mathrm{a}, \mathrm{in}}-T_{\mathrm{w}, \text { in }}\right)^{2}}\right]^{1 / 2}
$$

For Case A with $T_{\mathrm{a}, \text { in }}-T_{\mathrm{a} \text {,out }}=16.5 \mathrm{~K}, T_{\mathrm{a}, \text { in }}-T_{\mathrm{w}, \text { in }}=35 \mathrm{~K}$ and $\delta T=0.2 \mathrm{~K}$, the value of the relative uncertainty for $E$ is $1.9 \%$ at $r=10$.

The uncertainties in the water-air ratio are mainly associated with the volume flow rates of the spraying water and wetted air. The measuring accuracy of the used rotor flowmeter is $\pm 4 \%$. For Case A with $\delta Q_{\mathrm{v}, \mathrm{a}}=4.05 \mathrm{~m}^{3} / \mathrm{h}, Q_{\mathrm{v}, \mathrm{a}}=41.7 \mathrm{~m}^{3} / \mathrm{h}$ at $r=10$, the relative uncertainty of $r$ is $\frac{\delta r}{r}=\left[\left(\frac{\delta Q_{\mathrm{v}, \mathrm{a}}}{Q_{\mathrm{v}, \mathrm{a}}}\right)^{2}+\left(\frac{\delta Q_{\mathrm{v}, \mathrm{w}}}{Q_{\mathrm{v}, \mathrm{w}}}\right)^{2}\right]^{1 / 2}=10.5 \%$.

\section{Experimental results and discussion}

In this section, the heat transfer effectiveness and temperature rise of spraying water for different cases with the reference designs will be first analyzed. Then the heat transfer effectiveness for different cases with changed designs will be analyzed in detail.

To analyze the heat recovery performance of the above cases, two control factors, i.e., the water-air ratio and the inlet air temperature that affect the heat transfer effectiveness and the temperature rise of the spraying water will be taken into account. The temperature rise of the spraying water is defined as: $\Delta T=T_{\mathrm{w}, \text { out }}-T_{\mathrm{w}, \text { in }}$, which denotes the application value of the heated water. The inlet water temperature is fixed at $297 \mathrm{~K}$ during the experiment. As a reference case, $T_{\mathrm{a}, \text { in }}=333 \mathrm{~K}$. When the water-air ratio is varied, the flow rate of the spraying water remains constant.

\subsection{Heat transfer effectiveness and temperature rise of spraying water for different cases with reference designs}

As mentioned above, Case A is an empty tower, which is taken as the control group. 
In case B, 15 conical baffles are placed in the tower, and the geometric parameters of the conical baffle are shown in Fig. 3. In case C, 7 falling film plates with a thickness of $1 \mathrm{~mm}$ and several triangular water distributors were uniformly installed in the tower. Case D is an improved case based on Case C, where several baffle plates are installed on the falling film plate with an angle of $30^{\circ}$, as shown in Fig. 5. shows The effects of water-air ratio and inlet air temperature on the heat transfer effectiveness for four different cases with reference designs is illustrated in Fig.6.

From Fig. 6(a), one can find clearly that the increase of the water-air ratio can increase the heat transfer effectiveness for all cases. The maximum relative difference between the experimental points and the fitted trend curves are $4.9 \%, 2.0 \%, 3.3 \%$ and $3.2 \%$ for Case A, Case B, Case C and Case D, respectively. When $r>20$, the heat transfer effectiveness nearly remain constant, i.e., for Case A, Case B, Case C and Case $\mathrm{D}$, the values of heat transfer effectiveness are about $0.63,0.79,0.69$ and 0.77 , respectively. Hence, $r=20$ will be adopted in the following discussions. The values of $E$ of Case B, Case C and Case D are $25.5 \%, 9.5 \%$ and $21.8 \%$ respectively higher than that of Case A at $r=20$. The reason for the larger value of $E$ of Case B, Case C and Case D could be that the conical baffle, the falling plate and the baffle plate increase the flow resistance of the spraying water, and hence the contact time of the spraying water and the wetted air increases. Case B has the highest heat transfer effectiveness. The reason is that the conical baffle disturbs the wetted air flow in the tower and enhances the mass transfer between the wetted air and the spraying water. The heat transfer effectiveness of Case D is higher than that of Case C due to the effect of the 
baffle plate on the mass transfer between the wetted air and the spraying water. From Fig. 6(b), one can find that with the increase of the inlet air temperature, the heat transfer effectiveness decreases. The reason is that with the increase of the inlet air temperature, the heat transfer and the temperature difference of the inlet and outlet temperatures increases. However, the temperature difference is lower than the difference of the inlet air temperature and the inlet water temperature.

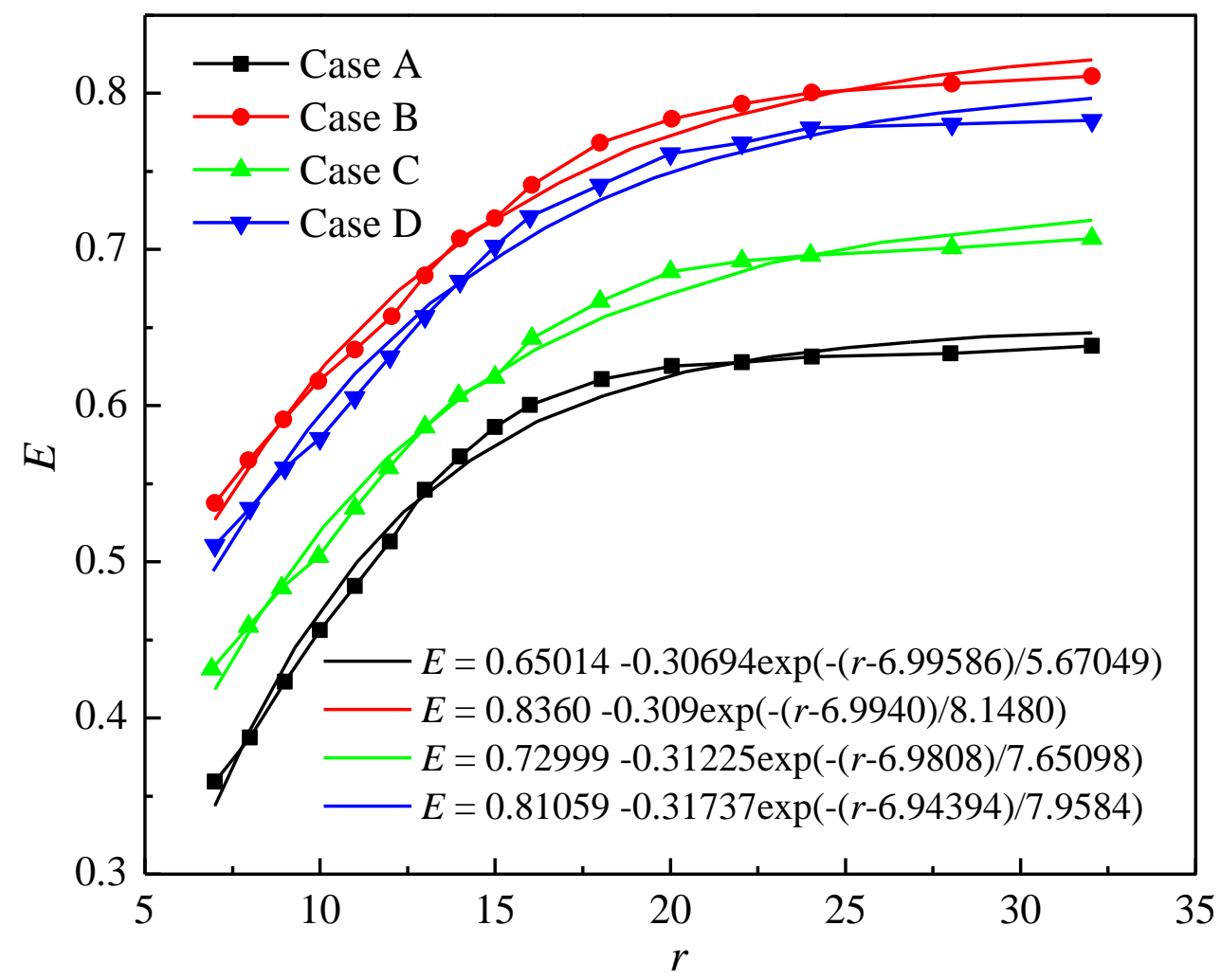

(a) water-air ratio 


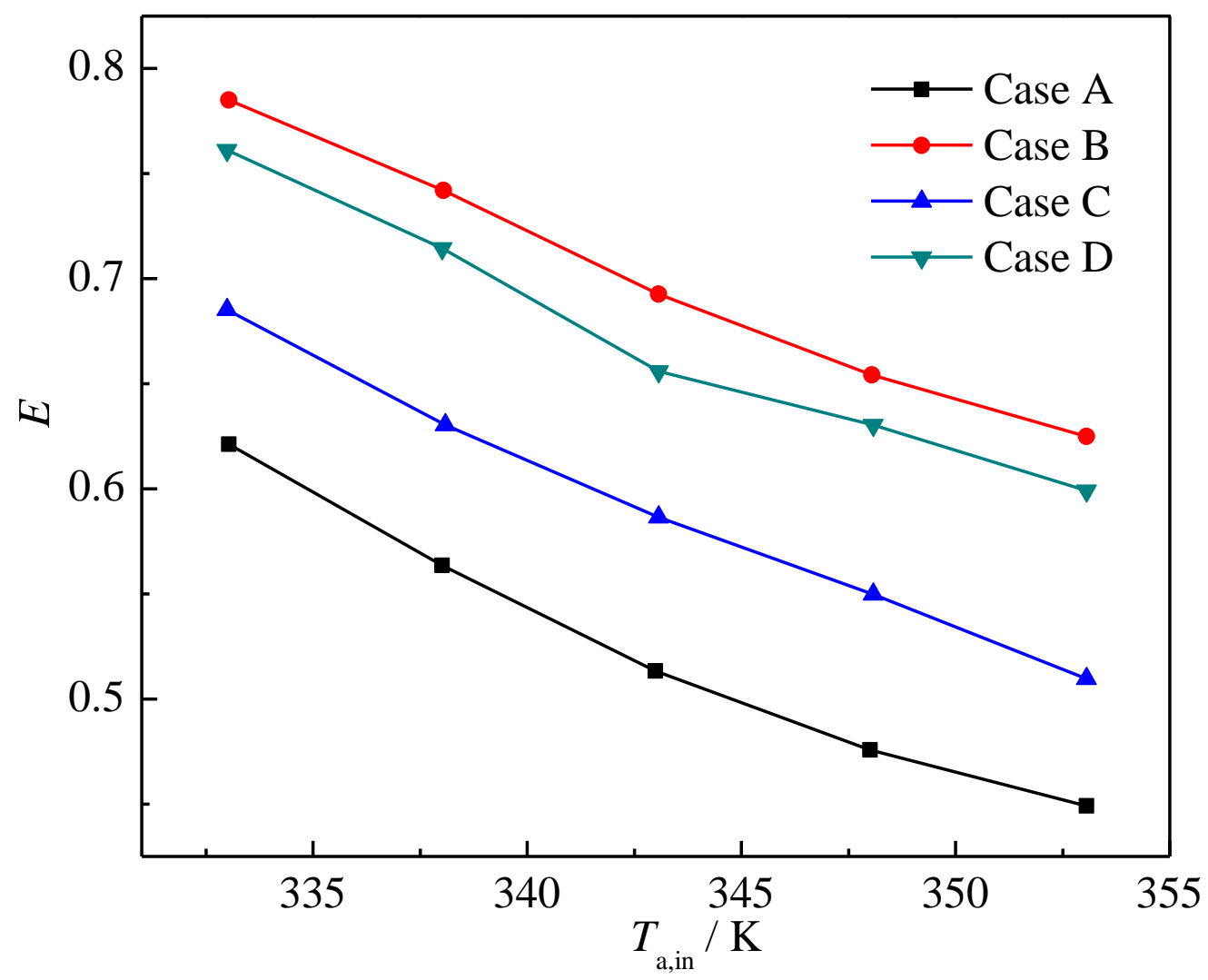

(b) inlet air temperature

Fig. 6 Effects of water-air ratio and inlet air temperature on heat transfer effectiveness for different cases.

Fig. 7 shows the effects of water-air ratio and inlet air temperature on the temperature rise of spraying water. From Fig. 7(a), the plot shows an initial sharp decrease of $\Delta T$ to a point $r=20$. This trend can be attributed to the increasing spraying water. From there till the end the $\Delta T$ decreases gradually. With the increase in the water-air ratio, $\Delta T$ for different cases becomes smaller. For example, the values of $\Delta T$ for Case A, Case B, Case C and Case D are $6.43 \mathrm{~K}, 7.87 \mathrm{~K}, 6.85 \mathrm{~K}$ and $7.34 \mathrm{~K}$, respectively, at $r=7$, meanwhile, the values of $\Delta T$ for Case A, Case B, Case C and Case D are $2.15 \mathrm{~K}, 2.35$ $\mathrm{K}, 2.26 \mathrm{~K}$ and $2.30 \mathrm{~K}$, respectively, at $r=20$. From Fig. 7(b), one can find that $\Delta T$ increases gradually with the increase of the inlet air temperature due to the increasing 
heat transfer. The larger values of $\Delta T$ and $E$ mean the higher quality of the heated water and the higher heat recovery performance. However, Fig. 7 also shows that $\Delta T$ and $E$ have the opposite tendency with the water-air ratio or the inlet air temperature. In order to obtain effective $\Delta T$ for practical application, the water-air ratio and the inlet air temperature should be optimized. In the following sections, the heat transfer effectiveness but not the temperature rise of spraying water will be discussed.

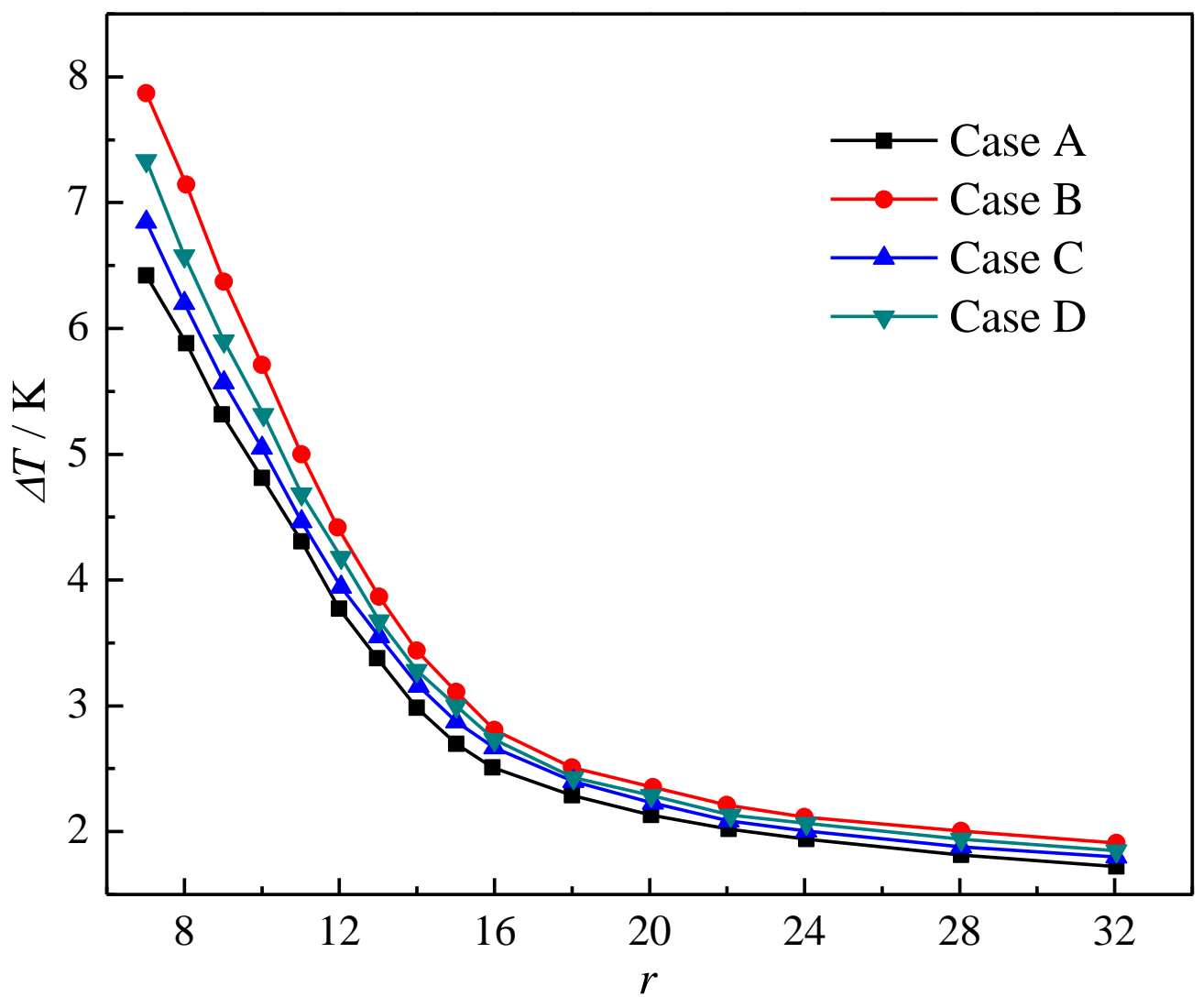

(a) water-air ratio 


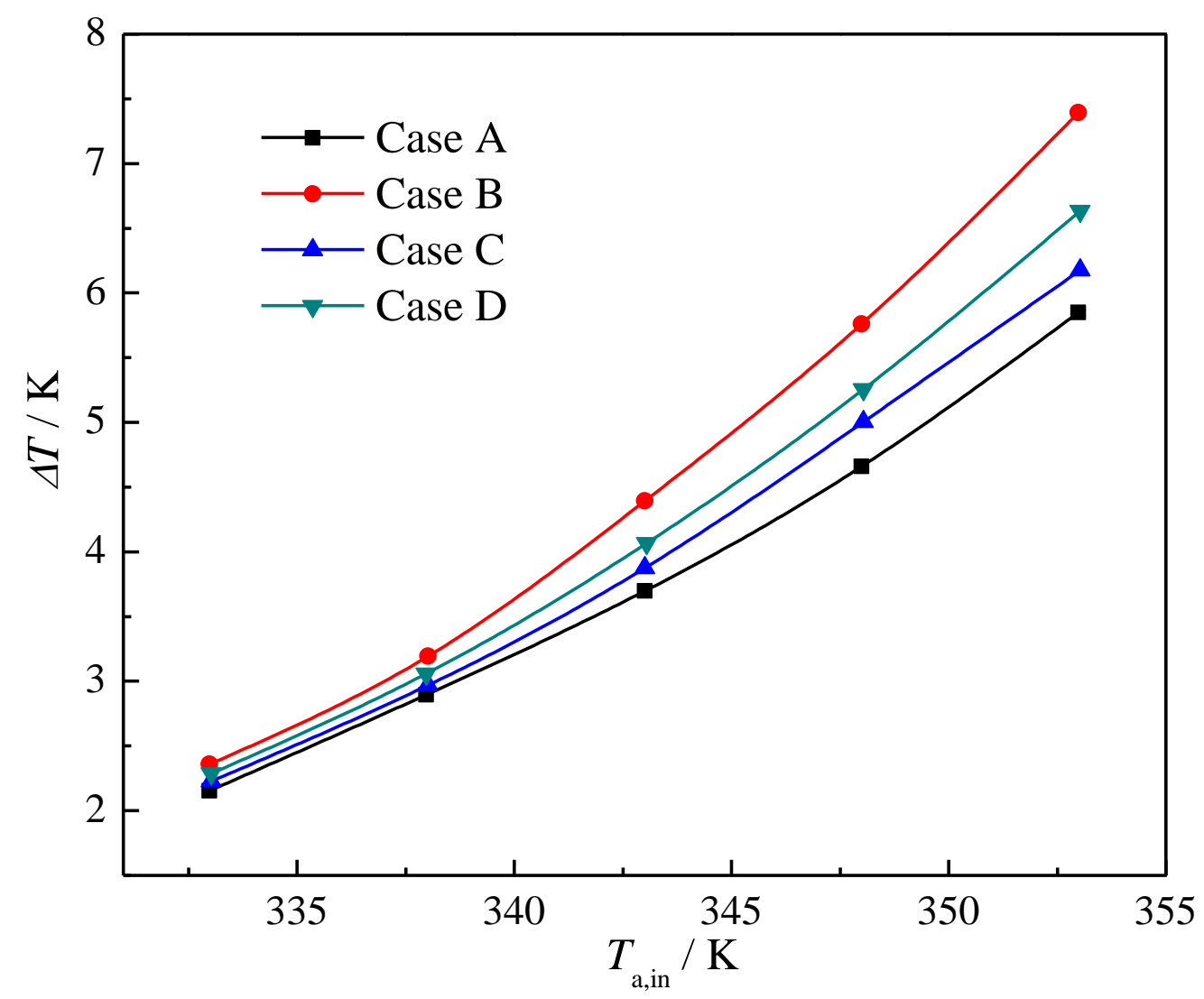

(b) inlet air temperature

Fig. 7 Effects of water-air ratio and inlet air temperature on temperature rise of spraying water.

\subsection{Heat transfer effectiveness for changed designs of different cases}

For both Case C and Case D, one of the main factors that affect the heat transfer effectiveness is the number of falling film plates, $n_{\mathrm{p}}$. Fig. 8 shows the heat transfer effectiveness with $n_{\mathrm{p}}=1,3,5$ and 7 for both Case C and Case D. From Fig. 8(a), for Case $\mathrm{C}$, the maximum relative difference between the experimental points and the fitted trend curves are $3.6 \%, 3.6 \%, 2.4 \%$ and $3.3 \%$ at $n_{\mathrm{p}}=1,3,5$ and 7 , respectively. For $n_{\mathrm{p}}$ $=1,3,5$ and 7, the values of $E$ are $0.64,0.67,0.70$ and 0.68 , respectively, at $r=20$. The values of $E$ at $n_{\mathrm{p}}=1,3,5$ and 7 for Case $\mathrm{C}$ are $2.4 \%, 6.9 \%, 12.8 \%$ and $9.5 \%$ respectively higher than that for Case A at $r=20$. From Fig. 8(b), for Case D, the maximum relative 
difference between the experimental points and the fitted trend curves are 3.5\%, $2.8 \%$, $2.3 \%$ and $6.6 \%$ at $n_{\mathrm{p}}=1,3,5$ and 7 , respectively. For $n_{\mathrm{p}}=1,3,5$ and 7 , the values of $E$ are $0.66,0.70,0.80$ and 0.76 , respectively, at $r=20$. The values of $E$ at $n_{\mathrm{p}}=1,3,5$ and 7 for Case D are 5.4\%, $12.3 \%, 28.3 \%$ and $21.8 \%$ respectively higher than that for Case A. It can be seen that with the increase of $n_{\mathrm{p}}$, the heat transfer effectiveness of both Case $\mathrm{C}$ and Case D increases. The maximum $E$ is obtained at $n_{\mathrm{p}}=5$. Especially, the heat transfer effectiveness of Case $\mathrm{D}$ at $n_{\mathrm{p}}=5$ is higher than that of Case $\mathrm{B}$ at reference design. The reason could be that both the cross-section area and the contact area between the water film and the wetted air increase with increasing of $n_{\mathrm{p}}$, and hence the heat transfer effectiveness increases. Whereas, too many falling film plates may lead to the larger shear stress of the wetted air on the water film, thereby disturbing the water film and decreasing the heat and mass transfer.

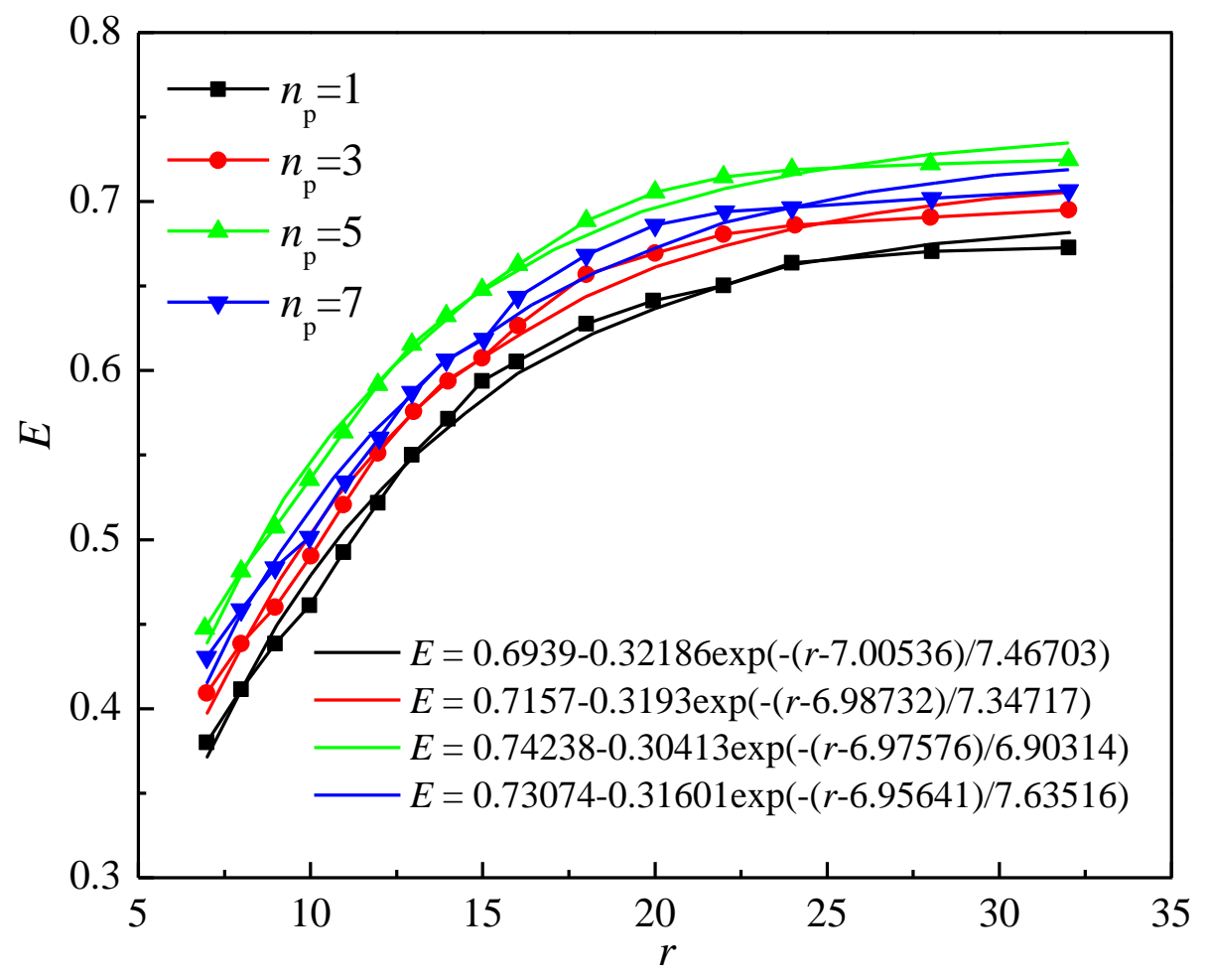

(a) Case C 


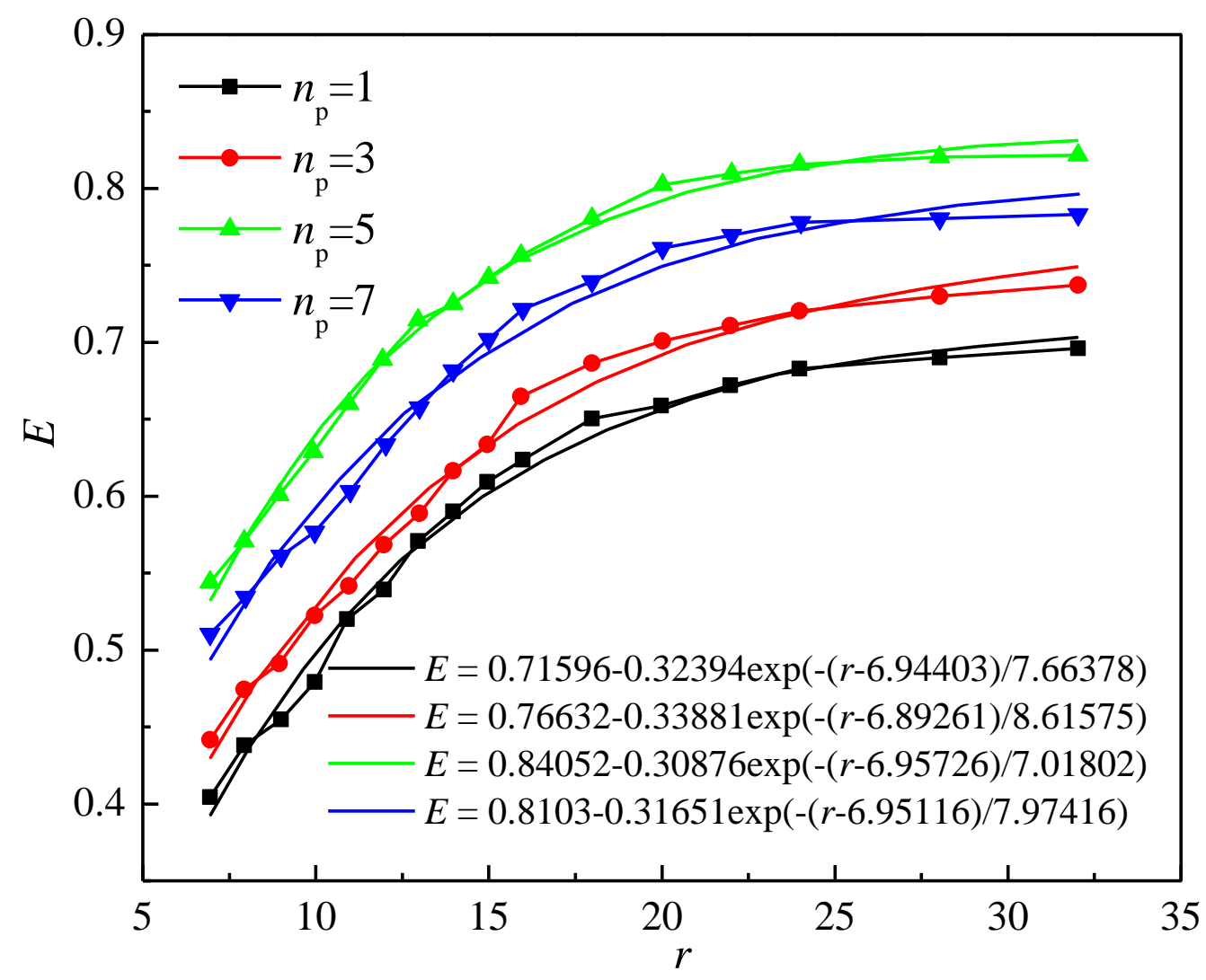

(b) Case D

Fig. 8 Effects of number of falling film plates on the heat transfer effectiveness for

Case C and Case D.

For Case B, the heat transfer effectiveness is affected by the number of conical baffles. In the current work, a new factor named the number of floors, $n_{\mathrm{f}}$, is defined in assisting the analysis. There are 8, 7, 8 and 7 baffles on the first, second, third and fourth floor from the top of the tower. The total number of the conical baffles is 15,23 and 30 for $n_{\mathrm{f}}=2,3$, and 4, respectively. Fig. 9 shows the heat transfer effectiveness with $n_{\mathrm{f}}=$ 2, 3 and 4 for Case B. The maximum relative difference between the experimental points and the fitted trend curves are $2.0 \%, 1.7 \%$ and $1.8 \%$ at $n_{\mathrm{f}}=2,3$ and 4 , respectively. $E=0.78,0.81$ and 0.85 at $n_{\mathrm{f}}=2,3$ and 4 , respectively at $r=20$. The values of $E$ at $n_{\mathrm{f}}=2,3$ and 4 for Case B are $25.5 \%, 30.4 \%$ and $36.6 \%$ respectively higher than 
that for Case A. The heat transfer effectiveness increases with the increase of $n_{\mathrm{f}}$. Especially, the heat transfer effectiveness of Case B at $n_{\mathrm{f}}=2$ and 3 is higher than that of Case $\mathrm{D}$ at $n_{\mathrm{p}}=5$. The reason is that the flow resistance and the contact area between the water and the wetted air obviously increase with the increasing of the number of the conical baffles.

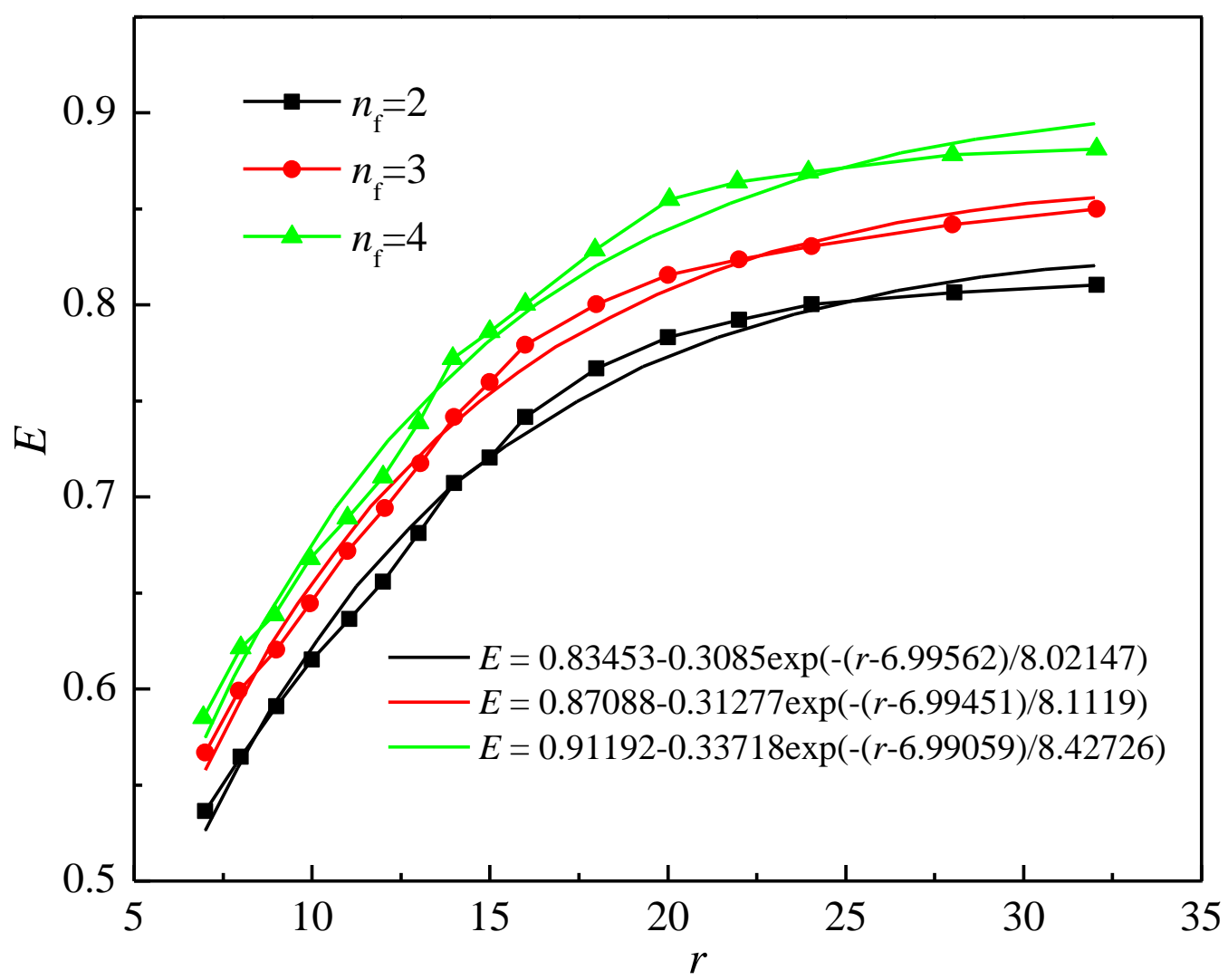

Fig. 9 The effects of the number of conical baffle floors on heat transfer effectiveness for Case B.

For all cases, the heat transfer effectiveness is affected by the central angle of the spiracles punched in the water sprayer. Now, the attention is turned to the effect of the central angle of the spiracle on the heat transfer effectiveness based on Case B with $n_{\mathrm{f}}$ $=4$, as shown in Fig. 10. The maximum relative difference between the experimental points and the fitted trend curves are $2.3 \%, 2.3 \%$ and $0.9 \%$ at $\alpha=30^{\circ}, 45^{\circ}$ and $60^{\circ}$, 
respectively. $E=0.85,0.86$ and 0.88 at $\alpha=30^{\circ}, 45^{\circ}$ and $60^{\circ}$, respectively at $r=20$. The values of $E$ at $\alpha=30^{\circ}, 45^{\circ}$ and $60^{\circ}$ for Case B are $36.6 \%, 39.0 \%$ and $41.0 \%$ respectively higher than that for Case A. The reason could be that the velocity of the water film is lower and the water film can be distributed more uniformly. In the whole designs, Case B with $n_{\mathrm{f}}=4$ and $\alpha=60^{\circ}$ shows the maximum heat transfer effectiveness, i.e., $E=0.88$.

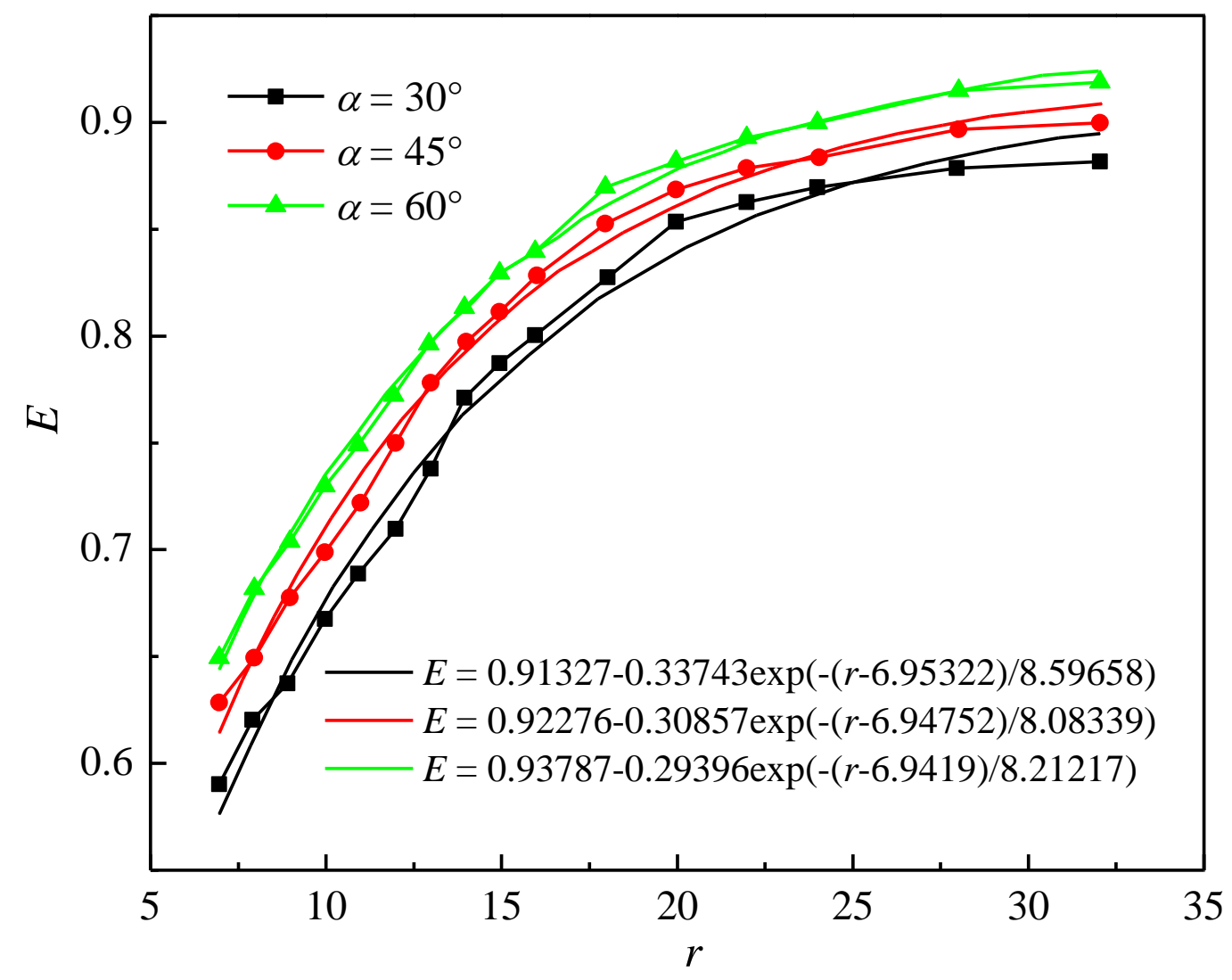

Fig. 10 The effects of the central angle of spiracle on the heat transfer effectiveness for Case B.

\section{Conclusions}

The falling film method was used for heat and moisture recovery from flue gas in the present work. Four experimental cases were presented and compared. The effect of several control factors such as water-air ratio, inlet air temperature, number of falling plates, number of conical baffle floors and central angle spiracles punched in the water 
sprayer on the heat recovery performance were examined. Analysis of the experimental results may lead to the following main conclusions:

(1) Increasing the water-air ratio or decreasing the inlet air temperature can improve the heat transfer effectiveness for different cases. For the case that the conical baffles staggered arranged in the tower has the highest heat transfer effectiveness. In comparison with the falling film plates, the arranged baffle plates can further improve the heat transfer effectiveness.

(2) For the cases with falling plates, the heat transfer effectiveness increases with the increase of the number of falling film plates. The maximum heat transfer effectiveness is obtained at 5 falling film plates. The heat transfer effectiveness of the cases with falling film plates and with baffle plates fixed on the falling film plate are $36.6 \%$ respectively higher than that of the empty tower.

(3) For the case with conical baffles, increasing the floor of the conical baffles can improve the heat transfer effectiveness. The heat transfer effectiveness of the case with 3 floors of conical baffles is $36.6 \%$ higher than that of the empty tower.

(4) In the range of the present work, the larger central angle of the spiracle is beneficial to increase the heat transfer effectiveness. For the case with 3 floors of conical baffles and $60^{\circ}$ central angle, the heat transfer effectiveness is $41.0 \%$ higher than that of the empty tower.

\section{Acknowledgement}

The present work is financially supported by the Natural Science Foundation of 
Hebei Province in China (20JCZDJC00470) and the National Natural Science Foundation of China (No. 51906059).

\section{Nomenclature}

\begin{tabular}{|c|c|c|c|}
\hline$d$ & diameter, $\mathrm{mm}$ & $\alpha$ & central angle of spiracles, \\
\hline$E$ & heat transfer effectiveness & $\theta$ & angle of baffle plate, ${ }^{\circ}$ \\
\hline$H$ & tower height, $\mathrm{mm}$ & $\Phi$ & diameter \\
\hline$L$ & tower length, $\mathrm{mm}$ & \multicolumn{2}{|c|}{ Subscripts and superscripts } \\
\hline$n$ & number & $\mathrm{a}$ & air \\
\hline$p$ & pitch of spiracles & $\mathrm{f}$ & floor \\
\hline$Q$ & flow rate & in & inlet \\
\hline$r$ & water-air ratio & $\mathrm{m}$ & mass \\
\hline$T$ & temperature, $\mathrm{K}$ & out & outlet \\
\hline$\Delta T$ & temperature difference, $\mathrm{K}$ & $\mathrm{p}$ & plate \\
\hline$W$ & tower width, mm & $\mathrm{V}$ & volume \\
\hline Gre & symbols & $\mathrm{w}$ & water \\
\hline
\end{tabular}

\section{References}

[1] J. Chai, Z. Wei, Y. Hu, S. Su, Z.G. Zhang, Is China's natural gas market globally connected, Energy Policy, 132 (2019) 940-949.

[2] BP Energy Outlook, 2019. Available from https://www.bp.com/content/dam/ bpcountry/zh_cn/Publications/19EOBook.pdf.

[3] M. Qu, O. Abdelaziz, H. Yin, New configurations of a heat recovery absorption 
heat pump integrated with a natural gas boiler for boiler efficiency improvement. Energy Convers. Manage. 87 (2014) 175-184.

[4] F.I. Abam, E.B. Ekwe, S.O. Effiom, M.C. Ndukwud, A comparative performance analysis and thermos-sustainability indicators of modified low heat organic Rankine cycles (ORCS): an exergy-based procedure, Energy Reports, 4 (2018) $110-118$.

[5] M.C. Barma, R. Saidur, S.M.A. Rahman, A. Allouhi, B.A. Akash, Sadiq M. Sait, A review on boilers energy use, energy savings, and emissions reductions, Renewable and Sustainable Energy Reviews, 79 (2017) 970-983.

[6] R. Vescovo, E. Spagnoli, High temperature ORC systems, Energy Procedia, 129 (2017) 82-89.

[7] H. Nami, I.S. Ertesvåg, R. Agromayor, L. Riboldi, L.O. Nord, Gas turbine exhaust gas heat recovery by Organic Rankine Cycles (ORC) for offshore combined heat and power applications energy and exergy analysis, Energy 165 (2018) 1060-1071.

[8] E.P.B. Júnior, M.D.P. Arrieta, F.R.P. Arrieta, C.H.F. Silva, Assessment of a Kalina cycle for waste heat recovery in the cement industry, Appl. Therm. Eng. 147 (2019) $421-437$.

[9] M. Mehrpooya, B. Ghorbani, S.A. Mousavi, Integrated power generation cycle (Kalina cycle) with auxiliary heater and PCM energy storage, Energy Convers. Manage. 177 (2019) 453-467.

[10]J. Wang, Y. Dai, L. Gao, Exergy analyses and parametric optimizations for different cogeneration power plants in cement industry, Appl. Energy 86 (2009) 941-948. 
[11]J.L. Hou, D.F. Che, Y.H. Liu, Q. Jiang, A new system of absorption heat pump Vs. boiler for recovering heat and water vapor in flue gas, Energy Procedia, 2018, 152: $1266-1271$.

[12]W. Salmi, J. Vanttola, M. Elg, M. Kuosa, R. Lahdelma, Using waste heat of ship as energy source for an absorption refrigeration system, Appl. Therm. Eng. 115 (2017) 501-516.

[13]L. Wang, G. Ma, A. Ma, F. Zhou, F. Li, Experimental study on the characteristics of triplex loop heat pump for exhaust air heat recovery in winter, Energy Convers. Manage. 176 (2018) 384-392.

[14]P. Zhang, T. Ma, W.-D. Li, G.-Y. Ma, Q.-W. Wang, Design and optimization of a novel high temperature heat exchanger for waste heat cascade recovery from exhaust flue gases, Energy 160 (2018) 3-18.

[15]Adéla Machác`ková, Radim Kocich, Marian Bojko, Lenka Kunc`ická, Krzysztof Polko, Numerical and experimental investigation of flue gases heat recovery via condensing heat exchanger, International Journal of Heat and Mass Transfer, 124 (2018) 1321-1333.

[16]Pan Zhang a, Ting Ma a, Wei-Dong Li b, Guang-Yu Ma b, Qiu-Wang Wang, Design and optimization of a novel high temperature heat exchanger for waste heat cascade recovery from exhaust flue gases condensing heat exchanger, Energy, 160 (2018) $3-18$.

[17]Guo Jiang-Feng, ZHANG Hai-Yan, CUI Xin-Ying, Huai Xiu-Lan, Han Zeng-Xiao, The Thermal Analysis of Low Grade Flue Gas Heat Recovery Heat Exchanger, Journal of Engineering Thermophysics, 1 (2020) 39-45.

[18]E. Tian, Y.L. He, W.Q.Tai, Research on a new type waste heat recovery gravity heat pipe exchanger, Appl. Energy 188 (2017) 586-594. 
[19]H. Jouhara, S. Almahmoud, A. Chauhan, B. Delpech, G. Bianchi, S.A. Tassou, R. Llera, F. Lago, J.J. Arribas, Experimental and theoretical investigation of a flat heat pipe heat exchanger for waste heat recovery in the steel industry, Energy 141 (2017) $1928-1939$.

[20]A. Akbari, S. Kouravand, G. Chegini, Experimental analysis of a rotary heat exchanger for waste heat recovery from the exhaust gas of dryer, Appl. Therm. Eng. 138 (2018) 668-674.

[21]R. Kong, T. Deethayat, A. Asanakham, T. Kiatsiriroat, Heat transfer phenomena on waste heat recovery of combustion stack gas with deionized water in helical coiled heat exchanger, Case Studies Therm. Eng. 12 (2018) 213-222.

[22]F. Li, L. Duanmu, L. Fu, X. Zhao, Research and application of flue gas waste heat recovery in cogeneration based on absorption heat-exchange, Procedia Eng. 146 (2016) 594-603.

[23]S. Zhao S. Yan, D.K. Wang, Y. Wei, H. Qi, T. Wu, P.H.M. Feron, Simultaneous heat and water recovery from flue gas by membrane condensation: Experimental investigation, Appl. Therm. Eng. 113 (2017) 843-850.

[24]D. Gao, Z. Li, H. Zhang, H. Chen, C. Cheng, K. Liang, Moisture and latent heat recovery from flue gas by nonporous organic membranes, Journal of Cleaner Production 225 (2019) 1065-1078.

[25]X. Quan, Y. Geng, P. Yuan, Z. Huang, C. Liu, Experiment and simulation of the shrinkage of falling film upon direct contact with vapor, Chem. Eng. Sci. 135 (2015) $52-60$. 
[26]L. Pu, Q. Li, X. Shao, L. Ding, Y. Li, Effects of tube shape on flow and heat transfer characteristics in falling film evaporation, Appl. Therm. Eng. 148 (2019) 412-419.

[27]M.W. Shahzad, M. Burhan, N. Ghaffour, K.C. Ng, A multi evaporator desalination system operated with thermocline energy for future sustainability, Desalination 435 (2018) 268-277.

[28]Zhu Xiao-Lei, Sui Xiao-Feng, Meng Ji-An, Li Zhi-Xin, Experimental Study on the Flow and Heat Transfer Characteristic of Saturated Moist Air - Water in Packing, Journal of Engineering Thermophysics, 37 (2016) 609-612.

[29]C. Qi, C. Min, S. Xie, X. Kong, Experimental study of fluid flow and heat transfer in a rectangular channel with novel longitudinal vortex generators, J. Enhan. Heat Transfer 17 (2010) 301-311. 